\title{
Synergistic antitumor activity of resveratrol and miR-200c in human lung cancer
}

\author{
TAO BAI, DAO-SONG DONG and LING PEI \\ Department of Anaesthesiology, The First Hospital of China Medical University, \\ Heping, Shenyang, Liaoning 110001, P.R. China
}

Received January 21, 2014; Accepted February 26, 2014

DOI: 10.3892/or.2014.3090

\begin{abstract}
MicroRNAs have emerged as promising molecular factors with potential for clinical applications in cancer diagnosis and therapy. In the present study, we demonstrated that the level of miR-200c in lung cancer tissues was lower than that in normal tissues using real-time PCR. To further investigate the effects of miR-200c expression in lung cancer cells, we upregulated miR-200c levels in H460 cells using transfection. We found that the percentage of apoptotic cells was higher in the cells expressing miR-200c than that in the untransfected cells. Furthermore, the antitumor activities of miR-200c were demonstrated in vivo. Notably, we confirmed that reservatol (RESV) showed stronger antitumor activities in miR-200c-positive cells than in miR-200c-negative cells. Finally, we demonstrated that expression of miR-200c in H460 cells suppressed cell growth by targeting RECK, followed by activation of the JNK signaling pathway and ER stress. Collectively, these data show that miR-200c expression sensitizes H460 cells to RESV and this is likely due to RECK expression.
\end{abstract}

\section{Introduction}

MicroRNAs (miRs) are evolutionary conserved small RNAs that modulate gene expression by inhibiting the protein translation process and/or degrading the respective target messenger RNA (1). In the past few years, microRNAs have emerged as promising molecular factors with potential for clinical applications in cancer diagnosis and therapy $(2,3)$. The miR-200 family of miRNAs consists of for five members (miR-200a, miR-200b, miR-200c, miR-141 and miR-429) expressed in two genomic clusters, one on chromosome 1p36.33 and the other on chromosome 12p12.31 (4). Recent evidence shows that miRNA-200 family members play a central role in the process of epithelial-mesenchymal transition (EMT) $(5,6)$. miR-200c

Correspondence to: Dr Tao Bai, Department of Anaesthesiology, The First Hospital of China Medical University, 155 Nanjing North Street, Heping, Shenyang, Liaoning 110001, P.R. China E-mail: baitao720418@163.com

Key words: resveratrol, miR-200c, lung cancer, apoptosis, RECK has been shown to promote the upregulation of E-cadherin by directly targeting the transcription factor ZEB1 (7). The importance of EMT in driving carcinogenesis has been shown in lung, breast, prostate, pancreatic and liver cancer $(8,9)$.

Resveratrol (RESV) (trans-3,4,5-trihydroxystilbene), a natural polyphenolic extracted from red wine, has the potential to inhibit the growth of several types of cancers such as prostate, lung and colorectal cancers (10-12). The role of miR-200c and RESV has been demonstrated in lung cancer, respectively; however, the synergistic antitumor activity of RESV and miR-200c remains unclear.

The aim of the present study was to identify potential roles and mechanisms of RESV and miR-200c in non-small cell lung cancer (NSCLC). Therefore, we specifically investigated i) the expression of miR-200c in the NSCLC cell line, H460, and its role in the proliferation of $\mathrm{H} 460$ cells; ii) the possible associations between miR-200c levels and RESV; and iii) the possible mechanisms involved in the enhancement of the antitumor activities of RESV by miR-200c.

\section{Materials and methods}

Subjects. Forty-two patients who were diagnosed with primary NSCLC at the Department of Thoracic Surgery, The First Affiliated Hospital of China Medical University from January 2006 to December 2010 were included in the present study. All diagnoses were based on standard laboratory tests (cytology and histology) and confirmed by computerized tomography of the thorax. None of the patients underwent radiotherapy or chemotherapy before the operation. The study was approved by the China Medical University Ethics Committee and was conducted by the Helsinki Declaration. All patients provided written informed consent to participate in the study.

H460 cell culture and transfection. The human non-small cell lung cancer cell line NCI-H460 was purchased from the American Type Culture Collection (ATCC; Manassas, VA, USA) and grown in RPMI-1640 culture medium containing $10 \%$ fetal bovine serum (FBS), 100 units/ml of penicillin, and $100 \mu \mathrm{g} / \mathrm{ml}$ streptomycin in humidified air $\left(5 \% \mathrm{CO}_{2}\right.$ atmosphere) at $37^{\circ} \mathrm{C}$. Cells were transfected with hsa-miR-200c (UAAUACUGCCGGGUAAUGAUGGA) (Open Biosystems, Huntsville, AL, USA) with RNAiFect transfection reagent (Qiagen, Gaithersburg, MD, USA). 
Real-time PCR. Total RNA was isolated from the cell lines and from lung specimens with TRIzol reagent (Invitrogen) according to the manufacturer's instructions. Expression of mature miR-200c was determined by the TaqMan miRNA assay (Applied Biosystems), and normalized using the $2^{-\Delta \Delta \mathrm{Ct}}$ method relative to U6. All TaqMan PCRs were conducted in triplicate with a fluorescence-based, real-time detection method (ABI PRISM 7500 Sequence Detection System; Applied Biosystems).

Resveratrol treatment and MTT assay. The transfected and untransfected cells were treated with RESV (Sigma Chemicals, St. Louis, MO, USA). MTT (10 $\mu \mathrm{l}$, at $5 \mathrm{mg} / \mathrm{ml})$ was added at a final concentration of $500 \mu \mathrm{g} / \mathrm{ml}$, and the mixture was further incubated for $1 \mathrm{~h}$ at $37^{\circ} \mathrm{C}$, and the liquid in the wells was removed thereafter. DMSO $(100 \mu \mathrm{l})$ was then added to each well, and the absorbance was read with a UVmax microplate reader (Molecular Devices, Sunnyvale, CA, USA) at $560 \mathrm{~nm}$.

Cell apoptosis assay. Cells $\left(5 \times 10^{5}\right)$ were collected without EDTA and washed with PBS. A $500 \mu 1$ binding buffer, $5 \mu 1$ Annexin V-FITC and $5 \mu \mathrm{l}$ propidium iodide (PI) were added into the suspension in that order and mixed at room temperature in the dark for $10 \mathrm{~min}$. The examination of apoptosis was performed by flow cytometry within $1 \mathrm{~h}$.

Cell cycle assay. Cells seeded on 6-well plates were treated and then collected. After being washed with PBS three times, the cell suspension was fixed with $70 \%$ ethanol, and incubated with RNAse A at $37^{\circ} \mathrm{C}$. After being stained with $400 \mu \mathrm{l} \mathrm{PI}$, the suspension was evaluated by flow cytometry.

Xenograft assays. All experiments using animals were performed according to the guidelines of the China Medical University Ethics Committee. Nude mice (nu/nu), 6-8 weeks of age, were purchased from Vital River Laboratories Co., Ltd. (Beijing, China). The animal facility was maintained under conditions of $20 \pm 2^{\circ} \mathrm{C}, 50 \pm 10 \%$ humidity, and a 12 -h light/dark cycle. $\mathrm{H} 460$ cells ( $3 \times 10^{7}$ in $\left.200 \mu \mathrm{l}\right)$ were subcutaneously injected into the axilla of each mouse. After the tumor diameters reached 3-5 $\mathrm{mm}$, the mice were divided randomly into three groups and received a $200 \mu \mathrm{l}$ intratumoral injection of miR-200c, RESV or both miR-200c and RESV. Two injections were administered at 10 a.m and 5 p.m. every two days. The tumor growth was then monitored for 30 days. Every five days until the end of the experiment, one mouse from each group was randomly selected to be sacrificed by $\mathrm{CO}_{2}$ asphyxiation, and tumors were harvested and weighed. The tissue was fixed in $4 \%$ paraformaldehyde for histopathologic analysis. Additional mice $(\mathrm{n}=60)$ were used to establish xenografts to obtain survival curves. Mice with xenografted tumors (as described above) that reached 3-5 $\mathrm{mm}$ in diameter were divided into four groups ( $\mathrm{n}=15$ for each). Survival was monitored until the experiments were terminated due to the heavy tumor burden.

miRNA target prediction. The miRNA targets predicted by TargetScan (http://www.targetscan.org/) are based on the presence of conserved $8 \mathrm{mer}, 7 \mathrm{mer}$ and $6 \mathrm{mer}$ sites that match the seed region of each miRNA (13). TargetScan also predicts
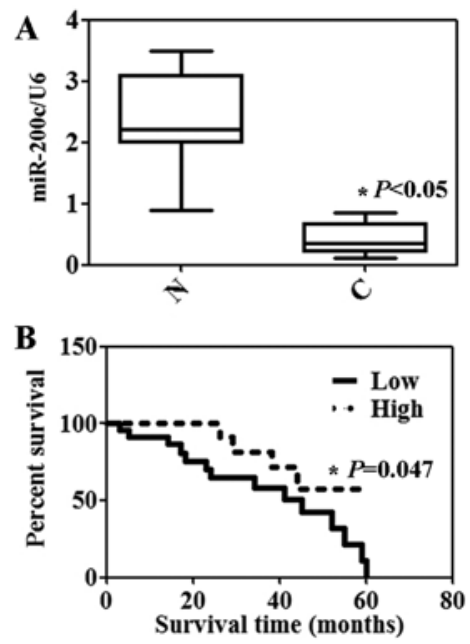

Figure 1. The level of miR-200c was measured in lung specimens using realtime PCR. (A) The level of miR-200c was lower in the cancer tissues than that in the matched normal tissues. U6 was used as an internal control. N, normal; C, cancer. (B) Kaplan-Meier curves of survival analysis indicating that patients carrying tumors with low miR-200c expression had poorer disease-specific survival than those with high miR-200c expression.

non-conserved sites, additional types of seed matches that are preferentially conserved in different species, and sites with mismatches in the seed region that are compensated by conserved 3' pairing (14).

Western blot analysis. Tissues and cells were lysed in lysis buffer $(20 \mathrm{mM}$ Tris-HCl, $150 \mathrm{mM} \mathrm{NaCl}, 2 \mathrm{mM}$ EDTA, $1 \%$ Triton X-100) containing a protease inhibitor cocktail (Sigma). Protein amounts were quantified using the BCA protein assay kit. Equivalent amounts of protein $(20 \mu \mathrm{g})$ were separated using $12 \%$ SDS-PAGE and transferred to a PVDF membrane (Millipore Corporation, Billerica, MA, USA). Western blot analysis was performed using primary antibodies: RECK (Santa Cruz Biotechnology, Santa Cruz, CA, USA), stress-activated protein kinase/JNK antibody (Cell Signaling Technology, Beverly, MA, USA), phospho-stress activated protein kinase/p-JNK (Thr $\left.{ }^{183} / \mathrm{Tyr}^{185}\right)$ (Cell Signaling Technology), CHOP (Abcam), BiP (Cell Signaling Technology), c-jun (Cell Signaling Technology), phosphorylated c-jun $\left(\operatorname{Ser}^{63}\right)$ (Cell Signaling Technology) and $\beta$-actin (Millipore). Binding of each specific antibody was detected with horseradish peroxidase (HRP)-conjugated respective secondary antibodies (Amersham Biosciences, Buckinghamshire, UK) and ECL solutions (Amersham Biosciences).

Statistical analysis. Data were analyzed using GraphPad Prism 5 software. Statistical analysis was performed using a one-tailed Student's t-test (unilateral and unpaired). KaplanMeier survival plots were generated, and comparisons between survival curves were carried out using the log-rank statistical analysis. P-values $<0.05$ were considered to indicate statistically significant differences.

\section{Results}

miR-200c is correlated with the 5-year survival rate of the patients with lung cancer. The level of miR-200c in the tumor 

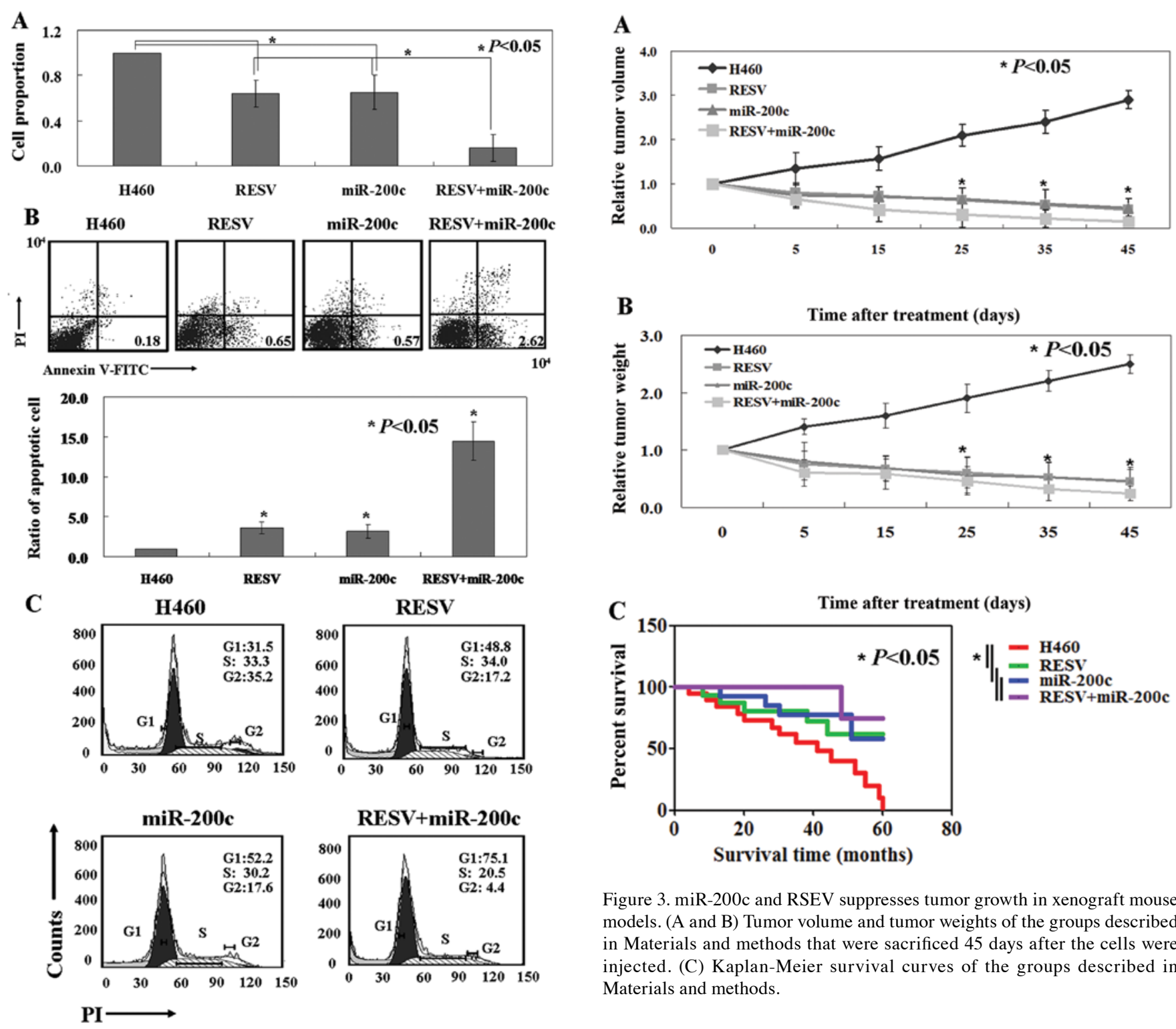

Figure 3. miR-200c and RSEV suppresses tumor growth in xenograft mouse models. (A and B) Tumor volume and tumor weights of the groups described in Materials and methods that were sacrificed 45 days after the cells were injected. (C) Kaplan-Meier survival curves of the groups described in Materials and methods.

Figure 2. Antitumor activities of miR-200c and/or RSEV in H460 cells. (A) Growth curve of the cell lines following the various treatments was measured using MTT assay. (B) The ratios of apoptotic cells were analyzed following double staining with Annexin V/propidium iodide (PI). (C) PI staining showed changes in the cell cycle distribution.

tissues was lower than that in the normal tissues as determined by real-time $\mathrm{PCR}(\mathrm{P}<0.05$; Fig. 1A). To investigate the association of miR-200c with patient survival, the survival data from 42 patients with NSCLC were assessed. Kaplan-Meier analysis showed that miR-200c expression was closely correlated with the favorable prognosis of patients with NSCLC $(\mathrm{P}<0.05$; Fig. 1B).

Growth inhibitory effects of RESV in miR-200c-expressing $H 460$ cells. A slower growth rate of the miR-200c-expressing $\mathrm{H} 460$ cells was noted when compared with the untreated cells by using MTT assay $(\mathrm{P}<0.05$; Fig. $2 \mathrm{~A})$. Annexin V/ PI double staining showed a higher level of apoptosis in the miR-200c-expressing $\mathrm{H} 460$ cells $(\mathrm{P}<0.05$; Fig. 2B). PI staining of cells revealed that miR-200c-expressing $\mathrm{H} 460$ cells were arrested in the $\mathrm{G}_{1}$ phase (Fig. 2C). RESV also inhibited prolif-

eration, induced apoptosis and $\mathrm{G}_{1}$ arrest in $\mathrm{H} 460$ cells (Fig. 2). Notably, RESV showed stronger antitumor activity in the miR200c-positive H460 cells than that in the negative miR-200c H460 cells. Furthermore, we confirmed that the activity of RESV in the miR-200c-positive H460 cells was not a simple summation of RESV and miR-200c (Fig. 2).

RESV and miR-200c inhibit tumor growth and prolong the survival rate of mice in vivo. We next determined whether RESV and miR-200c display antitumor activities in established xenograft tumor models. As shown in Fig. 3A, the tumor volumes of the RESV-treated and the miR-200c-treated mice were less than that of the untreated mice $(\mathrm{P}<0.05)$. Similarly, tumor weights for the RESV-treated group and the miR-200ctreated group were also significantly reduced when compared to that of the untreated group $(\mathrm{P}<0.05$; Fig. 3B). We also found that the RESV-treated and miR-200c-treated mice displayed a prolonged survival rate when compared to the untreated mice $(\mathrm{P}<0.05$; Fig. $3 \mathrm{C})$. The results of the in vitro studies demonstrated that the combined treatment with RESV and miR-200c had the strongest antitumor activity in the established xenograft tumor models. 


\begin{tabular}{|c|c|c|c|}
\hline $\begin{array}{l}\text { Target } \\
\text { gene }\end{array}$ & $\begin{array}{c}\text { Representative } \\
\text { transcript }\end{array}$ & & Gene name \\
\hline ZEB1 & \multicolumn{3}{|c|}{ NM_001128128 zinc finger E-box binding homeobox 1} \\
\hline FAM122C & \multicolumn{3}{|c|}{ NM_001170781 family with sequence similarity $122 \mathrm{C}$} \\
\hline DNA2 & \multicolumn{3}{|c|}{ NM_001080449 DNA replication helicase 2 homolog (yeast) } \\
\hline ZEB2 & \multicolumn{3}{|c|}{ NM_001171653 zinc finger E-box binding homeobox 2} \\
\hline SLC15A5 & \multicolumn{3}{|c|}{ NM_001170798 solute carrier family 15, member 5} \\
\hline LRP1B & NM_018557 & \multicolumn{2}{|c|}{ low density lipoprotein receptor-related protein 1B } \\
\hline SLC35B4 & NM_032826 & \multicolumn{2}{|c|}{ solute carrier family 35 , member B4 } \\
\hline WIPF1 & NM_001077269 & \multicolumn{2}{|c|}{ WASNASL interacting protein family, member 1} \\
\hline WNT16 & NM_016087 & \multicolumn{2}{|c|}{ wingless-type MMTV integration site family, member 16} \\
\hline ELL2 & NM_012081 & \multicolumn{2}{|c|}{ elongation factor, RNA polymerase II, 2} \\
\hline MCFD? & \multicolumn{3}{|c|}{ NM o01171506 multiple coagulation factor deficiency? } \\
\hline RECK & NM_021111 & \multicolumn{2}{|c|}{ reversion-inducing-cysteine-rich protein with kazal motifs } \\
\hline LEPR & \multicolumn{3}{|c|}{ NM_001003679 leptin receptor } \\
\hline SEC23A & NM_006364 & \multicolumn{2}{|c|}{ Sec23 homolog A (S. cerevisiae) } \\
\hline PROK2 & \multicolumn{3}{|c|}{ NM_001126128 prokineticin 2} \\
\hline \multicolumn{4}{|c|}{\begin{tabular}{|c|c|c|c|} 
& $\begin{array}{c}\text { predicted consequential pairing of target } \\
\text { region (top) and miRNA (bottom) }\end{array}$
\end{tabular}} \\
\hline \multicolumn{3}{|c|}{$\begin{array}{l}\text { Position 126-132 of RECK 3. UTR } \\
\text { hsa-miR-200c }\end{array}$} & 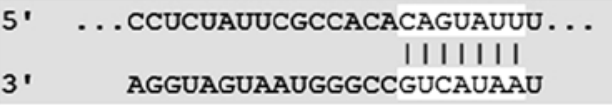 \\
\hline \multicolumn{3}{|c|}{ Position 1207-1214 of RECK 3. UTR } & 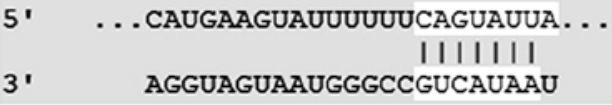 \\
\hline
\end{tabular}

Figure 4. The predicted molecular targets of miR-200c using TargetScan.

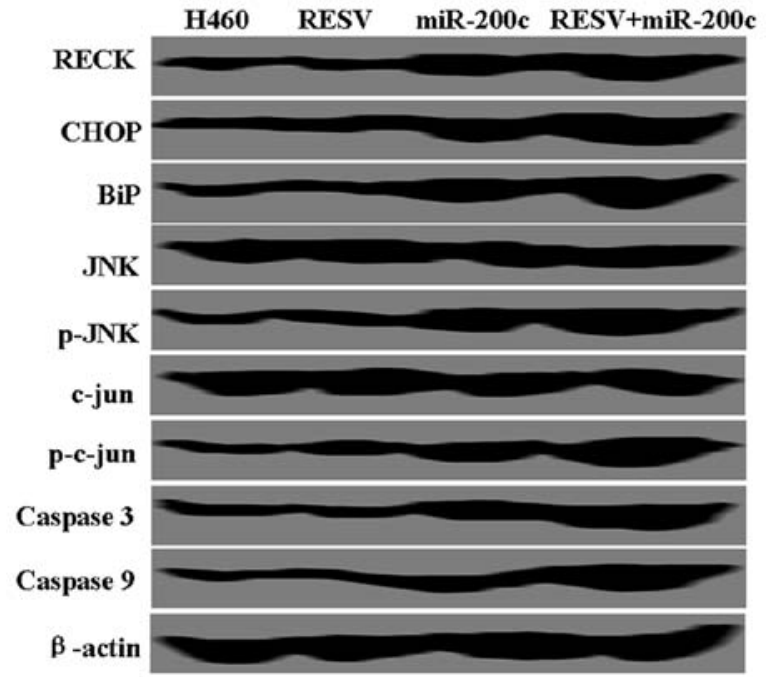

Figure 5. miR-200c suppresses H460 cell growth by targeting RECK. miR-200c exhibits antitumor activity by activating ER stress and JNK signaling pathway. RESV further enhanced RECK, BiP, CHOP, JNK, c-jun, caspase-3 and caspase-9 expression in the miR-200c-transfected cells but not in the untransfected ones. $\beta$-actin was used as an internal control.

Mechanism of RESV-induced apoptosis in the miR-200c-positive H460 cells. To clarify the mechanism of RESV in the miR-200c-positive H460 cells, TargetScan was used to deter- mine the predicted target genes of miR-200c. The prediction results are partly shown in Fig. 4. Furthermore, we focused on an ER stress-related protein, RECK. As shown in Fig. 5, RECK expression was upregulated following miR-200c transfection. Expression of ER stress molecules (BiP and CHOP) was significantly increased in the miR-200c-transfected cells. No significant changes in JNK and c-jun were detected in the transfected cells. However, phosphorylated JNK and c-jun were increased. We then confirmed that caspase-3 and caspase- 9 were activated in the transfected cells (Fig. 5). RESV further enhanced RECK, BiP, CHOP, JNK, c-jun, caspase-3 and caspase-9 expression in the miR-200c-transfected cells but not in the untransfected cells (Fig. 5).

\section{Discussion}

In the present study, we showed a highly significant correlation between relative miR-200c expression and the survival rate of the patients with NSCLC, indicating that its expression may be used as a valuable marker of lung cancer prognosis. Tang et al (15) also found that miR-200c was downregulated in gastric cancer specimens. Ceppi et al (16) confirmed that loss of miR-200c expression induces an aggressive, invasive, and chemoresistant phenotype in nonsmall cell lung cancer. Consistent with their studies, we found that miR-200c could induce apoptosis and $G_{1}$ arrest in H460 cells. Furthermore, we found that RESV exhibited 
a stronger antitumor activity in the miR-200c-positive $\mathrm{H} 460$ cells than that in the miR-200c-negative $\mathrm{H} 460$ cells.

The main aim of the present study was to determine the potential mechanism of RESV in miR-positive H460 cells. Firstly, we identified the regulation of miR-200c by potential molecular targets by using TargetScan, such as ZEB1 and ZEB2. Then we focused on reversion-inducing cysteine-rich protein with Kazal motifs (RECK), a membrane bound protein. Several studies have shown that RECK levels are significantly downregulated in human pancreatic, mammary, lung, prostate and colorectal cancers, as well as in hepatocarcinoma and neuroblastoma, when compared with the levels in the normal surrounding tissues (17-20). We confirmed that upregulation of miR-200c in H460 cells could increase RECK expression. The inhibitory role of RECK expression in cancer cells has been demonstrated both in previous (21) and in the present study. In addition to RECK expression, ER stress was detected in $\mathrm{H} 460$ cells. Chen et al (22) also found that RECK regulated endoplasmic reticulum stress response and enhanced cisplatininduced cell death in neuroblastoma cells. The alteration of the ER molecular chaperone GRP78/BiP expression levels can inhibit tumor growth in vivo (23). Another study also confirmed that ER stress-induced apoptosis is highly dependent on the upregulation of the UPR-inducible transcription factor CHOP (24). Consistent with previous studies, we found that the levels of BiP and CHOP in miR-200c-positive H460 cells were higher than levels in the untreated cells.

This is the first study to explore the role of miR-200c in controlling RECK expression in H460 cell lines. Our data showed that miR-200c expression sensitizes H460 cells to RESV, and this is likely due to RECK expression.

\section{Acknowledgements}

We thank Dr Ming-Yu Li for carefully proofreading the manuscript and for providing valuable comments.

\section{References}

1. Ryan BM, Robles AI and Harris CC: Genetic variation in microRNA networks: the implications for cancer research. Nat Rev Cancer 10: 389-402, 2010.

2. Kasinski AL and Slack FJ: MicroRNAs en route to the clinic: progress in validating and targeting microRNAs for cancer therapy. Nat Rev Cancer 11: 849-864, 2011.

3. Edwards JK, Pasqualini R, Arap W and Calin GA: MicroRNAs and ultraconserved genes as diagnostic markers and therapeutic targets in cancer and cardiovascular diseases. J Cardiovasc Transl Res 3: 271-279, 2010.

4. Tryndyak VP, Beland FA and Pogribny IP: E-cadherin transcriptional down-regulation by epigenetic and microRNA-200 family alterations is related to mesenchymal and drug-resistant phenotypes in human breast cancer cells. Int J Cancer 126: 2575-2583, 2009.

5. Korpal M,Lee ES,Hu G and Kang Y: The miR-200 family inhibits epithelial-mesenchymal transition and cancer cell migration by direct targeting of E-cadherin transcriptional repressors ZEB1 and ZEB2. J Biol Chem 283: 14910-14914, 2008.
6. Park SM, Gaur AB, Lengyel E and Peter ME: The miR-200 family determines the epithelial phenotype of cancer cells by targeting the E-cadherin repressors ZEB1 and ZEB2. Genes Dev 22: 894-907, 2008

7. Hurteau GJ, Carlson JA, Roos E and Brock GJ: Stable expression of miR-200c alone is sufficient to regulate TCF8 (ZEB1) and restore E-cadherin expression. Cell Cycle 8: 2064-2069, 2009.

8. Hugo H, Ackland ML, Blick T, et al: Epithelial-mesenchymal and mesenchymal-epithelial transitions in carcinoma progression. $\mathrm{J}$ Cell Physiol 213: 374-383, 2007.

9. Lee TK, Poon RT, Yuen AP, et al: Twist overexpression correlates with hepatocellular carcinoma metastasis through induction of epithelial-mesenchymal transition. Clin Cancer Res 12: 5369-5376, 2006.

10. Miki H,Uehara N, Kimura A, et al: Resveratrol induces apoptosis via ROS-triggered autophagy in human colon cancer cells. Int J Oncol 40: 1020-1028, 2012.

11. Sheths S, Jajoo S, Kaur T, et al: Resveratrolreducesprostate cancer growth and metastasis by inhibiting the Akt/ microRNA-21 pathway. PLoS One 7: e51655, 2012.

12. Yin HT, Tian QZ, Guan L, Zhou Y, Huang XE and Zhang H: In vitro and in vivo evaluation of the antitumor efficiency of resveratrol against lung cancer. Asian Pac J Cancer Prev 14: 1703-1706, 2013

13. Lewis BP, Burge CB and Bartel DP: Conserved seed pairing, often flanked by adenosines, indicates that thousands of human genes are microRNA targets. Cell 120: 15-20, 2005.

14. Jan CH, Friedman RC, Ruby JG and Bartel DP: Formation, regulation and evolution of Caenorhabditis elegans 3'UTRs. Nature 469: 97-101, 2011.

15. Tang H, Deng M, Tang Y, et al: $\mathrm{miR}-200 \mathrm{~b}$ and miR-200c as prognostic factors and mediators of gastric cancer cell progression. Clin Cancer Res 19: 5602-5612, 2013.

16. Ceppi P, Mudduluru G, Kumarswamy R, et al: Loss of miR-200c expression induces an aggressive, invasive, and chemoresistant phenotype in non-small cell lung cancer. Mol Cancer Res 8: 1207-1216, 2010.

17. Furumoto K, Arii S, Mori A, et al: RECK gene expression in hepatocellular carcinoma: correlation with invasion-related clinicopathological factors and its clinical significance. Reverseinducing-cysteine-rich protein with Kazal motifs. Hepatology 33: 189-195, 2001.

18. Span PN, Sweep CG, Manders P, et al: Matrix metalloproteinase inhibitor reversion-inducing cysteine-rich protein with Kazal motif a prognostic marker for good clinical outcome in human breast carcinoma. Cancer 97: 2710-7215, 2003.

19. Masui T, Doi R, Koshiba T, et al: RECK expression in pancreatic cancer: its correlation with lower invasiveness and better prognosis. Clin Can Res 9: 1779-1784, 2003.

20. Takeuchi T, Hisanaga M, Nagao M, et al: The membraneanchored matrix metalloproter nase (MMP) regulator RECK in combination with MMP-9 serves as an informative prognostic indicator for colorectal cancer. Clin Cancer Res 10: 5572-5579, 2004.

21. Lei L, Huang Y and Gong W: Inhibition of miR-92b suppresses nonsmall cell lung cancer cells growth and motility by targeting RECK. Mol Cell Biochem 387: 171-176, 2013.

22. Chen Y, Tsai YH and Tseng SH: RECK regulated endoplasmic reticulum stress response and enhanced cisplatin-induced cell death in neuroblastoma cells. Surgery 154: 968-979, 2013.

23. Moenner M, Pluquet $\mathrm{O}$, Bouchecareilh M, et al: Integrated endoplasmic reticulum stress responses in cancer. Cancer Res 67: 10631-10634, 2007.

24. Tamaki N, Hatano E, Taura K, et al: CHOP deficiency attenuates cholestasis-induced liver fibrosis by reduction of hepatocyte injury. Am J Physiol Gastrointest Liver Physiol 294: G498-G505, 2008. 